\title{
Identifying the business and social networks in the domain of production by merging the data from heterogeneous Internet sources
}

\author{
Dominik Kozjek $^{\mathrm{a}}$, Rok Vrabiča ${ }^{\text {, Gregor Eržen }}{ }^{\mathrm{a}}$, Peter Butala ${ }^{\mathrm{a}}$ \\ ${ }^{a}$ University of Ljubljana, Department of Manufacturing Systems and Control, Aškerčeva \\ 6, SI-1000 Ljubljana, Slovenia
}

\begin{abstract}
People, companies, and institutions form networks as part of their technical, economic, and social activities. As a consequence, these networks have an influence on how companies conduct business. Recently, the Internet, professional, and scientific social networks have contributed to the ease and simplicity of the network forming process and the public availability of the corresponding data. We investigate whether useful information about the relationships between individuals, companies, and institutions for the domain of production can be extracted from publicly available, structured and unstructured data that is merged from various Internet sources. We demonstrate that relevant information about the structures of these networks can be obtained by merging publicly available data using a combination of advanced computational methods including web crawling, machine learning, and creating mash-ups of publicly available services. The feasibility and the applicability of the approach are shown for a case in the automotive domain. A potential use case of the resulting data is demonstrated, showing how the approach can be used to find specific skills and expertise for a scientific
\end{abstract}


community consisting of people from industry and academia. The proposed approach can be used for the modelling and analysis of various forms of collaboration between and within businesses. As a tool, it could be used for the purposes of strategic networking, to facilitate the creation of project consortia, to identify competitors and other stakeholders in a certain domain, to pinpoint communication channels, to search for specific expertise, or to identify organisational and social structures within organisations.

Keywords:

Production, Network, Network Analysis, Data Analytics, Skill Profiling

\section{Introduction}

Individuals and businesses form networks as part of their professional and social activities. Companies form networks in various ways and forms Wiendahl and Lutz, 2002), e.g., when they sign partnership agreements, engage suppliers, or enter project consortia (Papakostas et al., 2013). The inner workings of companies can also be considered as networks of departments and individual people. These networks are not only technical in nature, but are, besides having an organisational role, often underlined by social relationships and motives (Škulj et al., 2014; Putnik et al., 2015; Mourtzis et al., 2016a). Modelling the inter- and intra-company relations as networks and analysing their structure can be used as a powerful tool for the management of various aspects of business operations (Mourtzis et al., 2013; Lanza and Moser, 2014; Becker et al., 2014; Kito et al., 2015; Neto et al., 2016; Zhong et al., 2015; Acar and Atadeniz, 2015).

The Internet and, specifically, professional and scientific social networks 
have simplified the networking process and improved its accessibility. Individuals and companies alike are using online networks to maintain their realworld connections, to keep up with the achievements of their peers, and to share various types of information with the interested public. This has caused a growth in the unstructured, heterogeneous, and already large amounts of data, which is of low density, but highly valuable, and which can be merged, aggregated, and analysed in order to extract the valuable information that is buried within it (Chae, 2015).

The emergence of open, publicly accessible data allows people, companies, and organisations to create data-driven services, analyse patterns and trends, make data-driven decisions, and solve complex problems. However, in order to harness the value of the data from unstructured sources such as the Internet, it has to be structured and linked in intricate ways.

This paper investigates whether useful information about the relationships between individuals, companies, and institutions from the domain of production can be extracted from publicly available, structured and unstructured data that is merged from various Internet sources. It demonstrates that this requires a combination of computational approaches and techniques, such as web crawling, heuristically guided searching, text extraction, data mining, and network analysis, which are used for the acquisition, validation, and analysis of the data.

The approach is applied to the case of OEM - supplier relations in the automotive sector in order to check whether known relations between companies are reflected. The approach is applied in the case study of a network consisting of professionals and scientists from the domain of production en- 
gineering, from both industry and academia, to demonstrate the use of the resulting data for skill and expertise profiling. Other uses of the approach are outlined.

\section{Managing data from heterogeneous Internet sources}

Businesses and individuals are ever more aware of the importance of having an online presence and visibility. By using the Internet, companies can increase their reach, promote their brand, and engage potential customers, while individuals can advertise their skills on the job market and pitch their research. Even though the motivations for increasing visibility are varied, the result is similar: the emergence of vast amounts of publicly available data that is unstructured, unreliable, and incomplete, and which changes over time. The data may possess one or more characteristic attributes related to its volume and complexity (Laney, 2001; Wamba et al., 2015): (1) volume, i.e., the amount of data, (2) velocity, i.e., the changeability of the data, (3) variety, i.e., the format of the data, (4) value, and (5) veracity, i.e., the accuracy of the data (Mourtzis et al., 2016b; Hitzler and Janowicz, 2013). Advanced data analysis is needed to achieve new insights by combining traditional approaches and alternative data analytics approaches such as probabilistic data structures, machine learning, and massively parallel computing (Hu et al. 2014, Chen et al., 2014).

Key challenges of applying the advanced computational methods to sources such as the Internet include devising a sufficiently powerful data structure, creating and applying methods that make sense of structured and unstructured data sources, as well as linking and merging data from different sources 
based on context and similarity (Tan et al. 2015). These challenges are addressed in the next section which describes the models and the computational methods used in the presented research.

\section{Identification of business and social networks in the domain of production}

Information is sought for three types of entities: people (individuals), companies, and institutions. These interact through several mechanisms, two of which are of interest: business- and socially motivated interactions. For example, business interactions include OEM - supplier relationships between companies, employer - employee relationships between companies and individuals, and research partnerships between institutions. Social interactions are considered to be all interactions amongst people. The objective is to identify how and to what extent these interactions are reflected on the Internet. For example, the websites of suppliers might have web links to the websites of the OEMs, peoples' profiles on social and professional network websites might include information about their current and past employments, and research collaborations between institutions might be visible through the co-authoring of scientific papers.

Not all of this information might be present in its entirety; it might not be publicly available; and it might not be feasible to extract some of the information given finite computational resources. Therefore, three main sources of information, chosen because they promise to describe the interactions in question, are selected:

- a web crawl of companies' and institutions' websites to identify links 
to other companies and institutions,

- a professional social network where all types of nodes are represented, and

- a scientific social network that mostly includes the profiles of research institutions and their employees.

Furthermore, the search through these sources is limited to the domain of interest, i.e., production engineering, in order to limit the scope of the investigation.

The entities and their interactions can be represented as networks in which nodes represent entities and connections represent the interactions. Fig. 1 presents an overview of the process of identifying the networks. Realworld networks consist of people, companies, institutions, and other nodes, i.e., nodes that are not in the domain of interest. Their profiles and the information about their interactions are sought through the three sources of information. The identified network is the representation of the real-world network, constructed through a process of searching for corresponding online websites and profiles, and identifying their interconnections.

The network is defined as a graph $G$ of nodes $N$ and edges $E$. $N$ is a set of $R$ nodes. $E$ is a set of $S$ edges. Each edge is defined as a 2-tuple of nodes. These relations are shown in Equations 1-3.

$$
\begin{gathered}
G=(N, E) \\
N=\left\{n_{1}, n_{2}, \ldots, n_{R}\right\}
\end{gathered}
$$




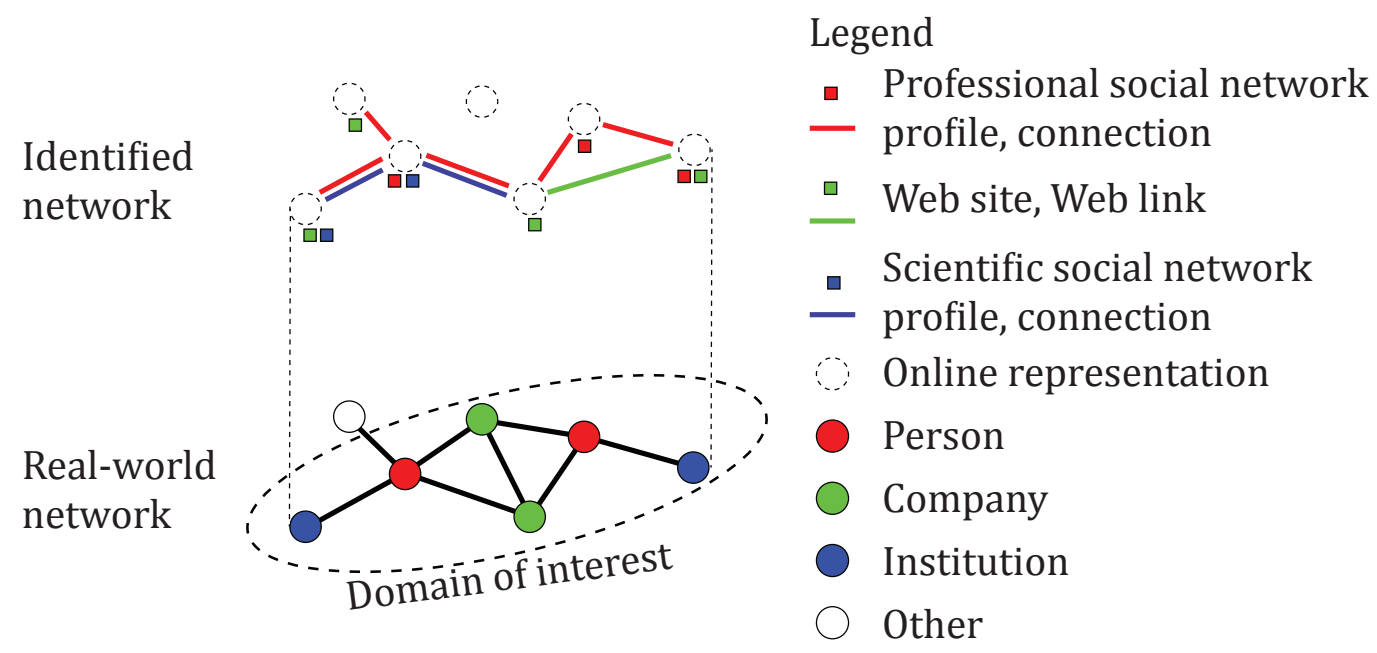

Figure 1: Overview of the network-identification process.

$$
E=\left\{e_{1}, e_{2}, \ldots, e_{S}\right\} ; \forall e \in E: e=\left(n_{i}, n_{j}\right)
$$

Nodes and edges have several properties, formally defined as associations between nodes or edges and the values of the property. Nodes are assigned a name, a URL addresses linking to the Internet websites associated with the node, a type, a domain, keywords describing the node, and a geographical location. The node name is defined as an association between the nodes $N$ and their names, which are strings $\mathbb{S}$.

$$
\text { name }: N \rightarrow \mathbb{S}
$$

URL addresses link nodes with their online representations, i.e., the websites, the social, and the professional network profiles. These are encoded as strings. There may be zero or more representations for each node. 


$$
\text { urls }: N \rightarrow \mathbb{S}^{u}
$$

The node type is defined as an association between a node and one of the four possible types: person $(P)$, company $(C)$, institution $(I)$ and other $(O)$, as shown in Equation 6 .

$$
\text { type }: N \rightarrow\{P, C, I, O\}
$$

Keywords describe each node in detail by associating them with strings, found on their online representations. This procedure is described in the following sections.

$$
\text { keywords }: N \rightarrow \mathbb{S}^{k}
$$

The geographical location of nodes is represented as latitude $\phi$ and longitude $\lambda$ in the standard geographic coordinate system. Both are real numbers.

$$
\text { geolocation }: N \rightarrow \mathbb{R} \times \mathbb{R}
$$

Edges have one property, i.e., the type of the edge, the value of which is a string.

$$
\text { edgetype }: E \rightarrow \mathbb{S}
$$

Shorthand notation, such as $n_{\text {type }}$ for node type, is used for the properties here on out. The lists of all node and edge properties are shown in Equations 10 and 11 . 


$$
\begin{gathered}
n=\left\{n_{\text {name }}, n_{\text {urls }}, n_{\text {type }}, n_{\text {domain }}, n_{\text {keywords }}, n_{\text {geolocation }}\right\} \\
e=\left\{e_{\text {edgetype }}\right\}
\end{gathered}
$$

The identification procedure is performed in a breadth-first manner and consists of five steps as shown in Fig. 2. The initialisation step (Step 1) is performed once at the beginning, while the other steps (Step 2-5) are repeated in cycles. In each cycle, all the connections of the existing nodes are identified and newly found nodes are added to the network. The cycle in which the node $n$ is identified is denoted as $i: n^{i}$.

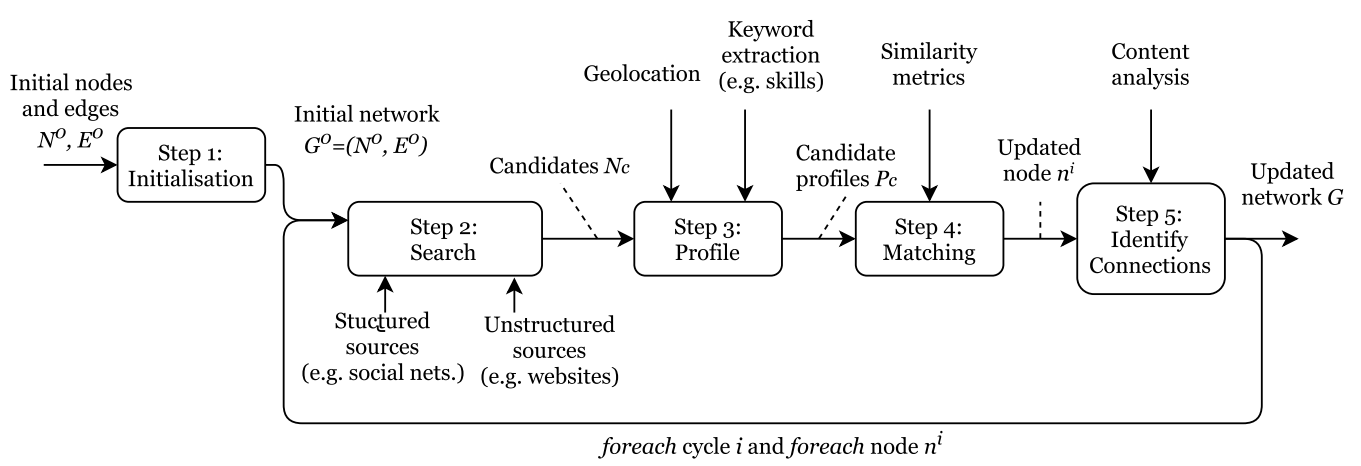

Figure 2: The steps of the network identification procedure.

\subsection{Step 1: Initialisation}

The seeds, i.e., the initial nodes for the search, are defined manually at the beginning. They can include any person, company, and/or institution. If known, additional information, such as the website, the social network profile, and/or the geographic location, can be provided. The type of the 
initial seed nodes $N^{0}$ can only be people $(\mathrm{P})$, companies $(\mathrm{C})$ or institutions (I).

$$
N^{0}=\left\{n^{0} \mid n_{\text {type }}^{0} \in\{P, C, I\}\right\}
$$

Initial edges $E^{0}$ can only be defined for pairs of seed nodes.

$$
E^{0}=\left\{e=\left(n_{1}^{0}, n_{2}^{0}, e_{\text {type }}\right):\left\{n_{1}^{0}, n_{2}^{0}\right\} \subseteq N^{0}\right\}
$$

The initial network graph is created from the seed nodes and initial edges $G^{0}=\left(N^{0}, E^{0}\right)$. The network identification process takes the initial network and the number of cycles as input parameters, and then extends the network in each cycle by searching for online representations of nodes (Step 2), profiling the results (Step 3), matching them with existing information (Step 4), and identifying new connections and nodes (Step 5). The process, described in pseudocode, is presented in Algorithm 1.

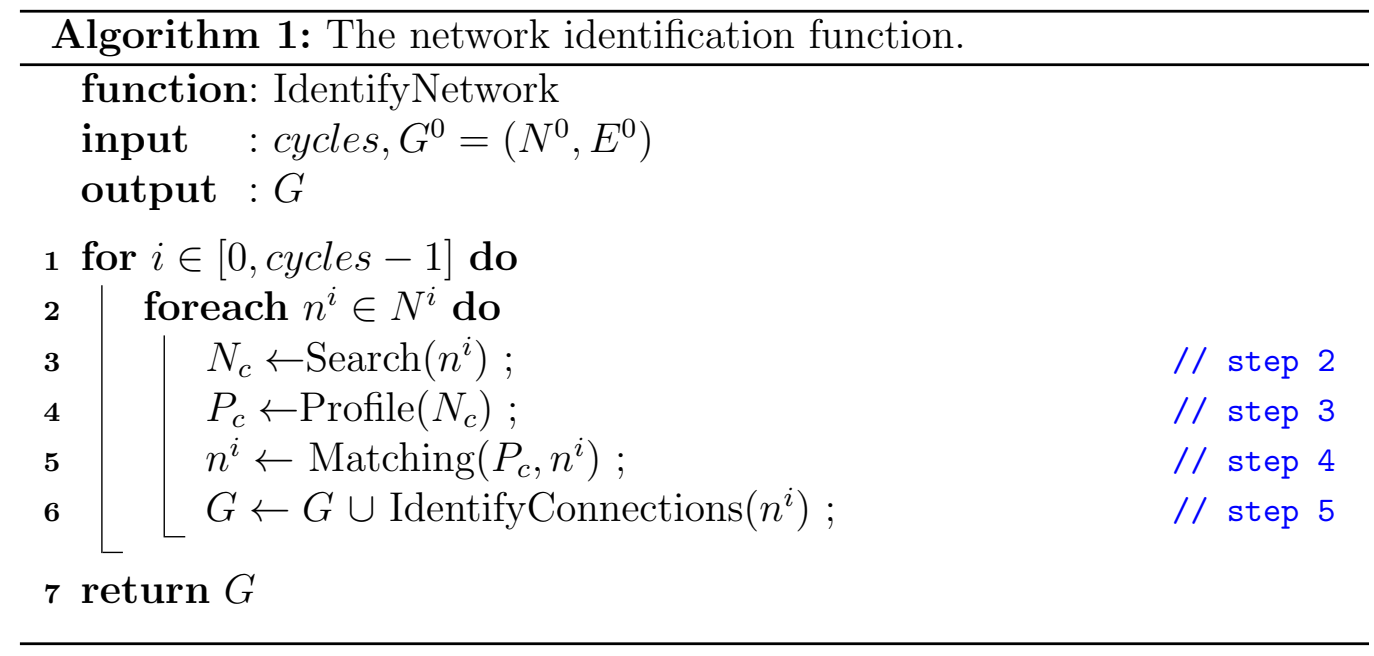

The steps 2-5 are described in the following subsections. 


\subsection{Step 2: Search}

The information sources are first searched for matching profiles based on existing incomplete information. The input for the search algorithm is the node $n^{i}$. The output is a set of candidates $N_{c}$.

In the case of the unstructured sources such as the web crawl, a search engine is used to find candidate websites for a company or an institution. The search engine string consists of the name and the country of the entity. For the structured sources such as social networks, either proprietary search mechanisms or a search engine are used to find the candidate profiles. The search engine string consists of the name of the social network, and the name and the country of the entity. Only structured sources are used when searching for people $(P)$, while both structured and unstructured sources are used for companies $(C)$ and institutions $(I)$. The result of the search is a set of candidate nodes (candidates) for which it is yet to be determined whether they represent the input node. In order to reduce the effort needed for further steps, candidate nodes with type different than the type of the input node $\left(n^{i}\right)$ are filtered immediately, as are nodes belonging to domains other than the domain of interest - production. The search function is presented in Algorithm 2,

A type and a domain are assigned to each candidate profile. In the case of social networks the type assignment is straightforward, based on the structure of the URL address and the textual information on the corresponding website. For the web crawl, however, the type assignment is nontrivial, because it has to be inferred from the text of the website. For this, a combination of several mechanisms is used: automatic detection of the language, translation of the 


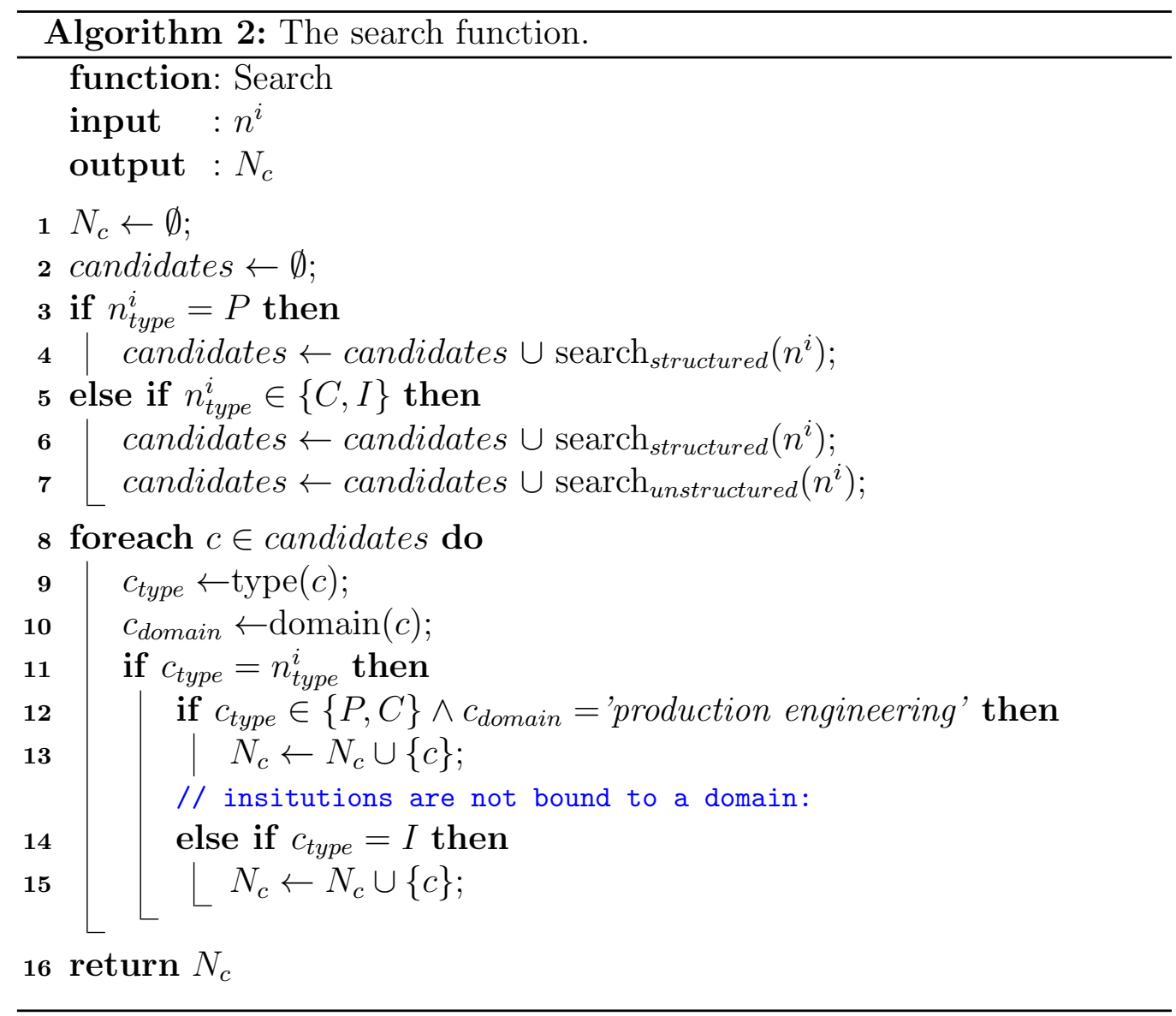

website's keywords into English, and keyword matching through which both the type and the domain are inferred.

\subsubsection{Determining the type and the domain of the node}

Websites are used, among other purposes, to describe the entity to which they belong. It is very easy for human beings to distinguish whether a website belongs to a known entity type, such as a company or an institution, based on the structure and the contents. However, automatically determining the type and the domain of the entity based on website contents proves to be 
difficult for computers. A promising approach is to use methods of machine learning in which a classifier is trained on training data for which the type and the domain are known in advance (Qi and Davison, 2009). In turn, the classifier is able to estimate the type and the domain of new, unknown data.

In the scope of this research, a keyword based multi-class classification model is built and evaluated. The rest of this section describes the training data, the model and its implementation, and the issues related to the approach such as multilingualism, limitations of available web services, and diversity of the data.

For the purpose of this research, the training data is gathered from freely accessible databases (IQS, 2016; Alexa, 2017) which provide categorised URL addresses of thousands of websites. 4300 websites were downloaded from manually selected categories representing (1) companies from the production domain, (2) institutions, and (3) others, such as search engines, video stream providers, social networks, etc. App. the same number of websites is chosen for each of the three categories.

In the preprocessing step, the textual contents are extracted for each of the websites. The term frequency is determined. Common stop words are ignored. App. twenty keywords which identify a category are chosen for each of the categories (e.g. "machine", "support", "product", "commerce", "tool", etc. for companies, and "university", "institute", "research", "alumni", "campus", etc. for institutions). The term frequency of the keywords and the known category are the input for the classification.

Due to the limitations of translation services and computational limitations, it is at the time not feasible to translate all text from all the websites. 
In order to deal with multilinguistic nature of the websites, dictionaries were built, translating the keywords into 40 languages.

The classification model is built with JRip algorithm (Rajput et al., 2011; Witten et al., 2016). The performance is validated with 10-fold cross validation. The classification accuracy is $77 \%$. Precision and recall which describe individual target class purity and misclassifications of instances are $74 \%$ and $79 \%$ respectively for companies, $84 \%$ and $91 \%$ for institutions, and $59 \%$ and $70 \%$ for others.

A URL filter is used to exclude some of the most popular websites, such as large social networks and video providers. The classification of a new website then consists of the following steps: (1) website data download, (2) extraction of contents (text), (3) language detection for purpose of choosing an appropriate dictionary of keywords, (4) keyword detection, and (5) classification using the keyword based classification model.

The presented approach for the classification of the type and the domain is fast and robust. The accuracy, which is approx. 80\%, proves to be good enough to retain the large-scale picture of the network structure.

\subsection{Step 3: Profiling}

In this step, detailed profiles of candidate nodes are generated. The candidates are geographically located, for which external services for geolocation, i.e., getting geographic coordinates from the physical address, and IP geolocation, i.e., getting geographic coordinates from the IP address, are used. The profile function is presented in Algorithm 3.

The detailed profile also includes keywords, extracted from the content of the candidate's website and social network profiles. The keyword extrac- 


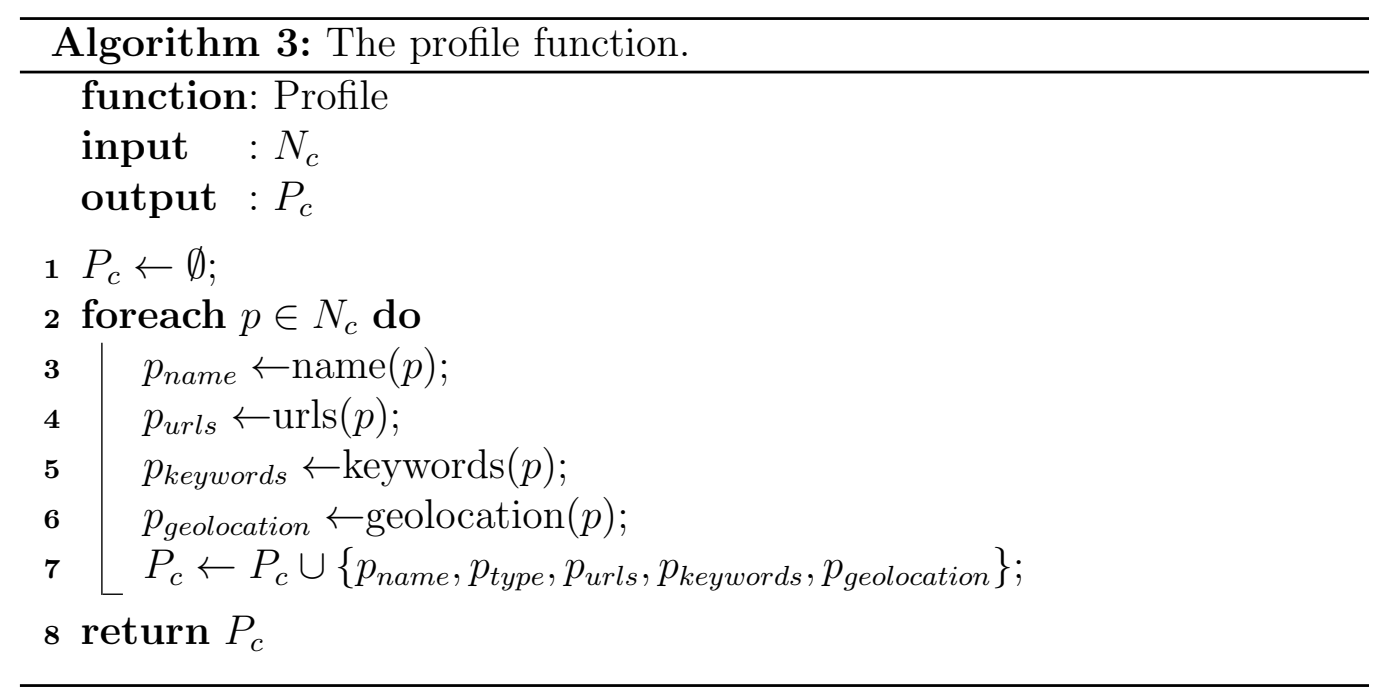

tion is straightforward: in case of websites, the contents of the website are matched agains a list of keywords from the domain of production engineering, while in the case of social network profiles where the keywords such as field of work, skills, and expertise are explicitly stated, they are extracted based on the structure of the website. An example profile is presented in Table 1.

\subsection{Step 4: Matching}

Each candidate profile is then matched to the existing node information. The matching is based on the similarity of the name, the URLs, and the location.

The similarity metric for textual data can be any string metric for measuring the difference between words. In the presented work, Levenshtein distance is used. The similarity metric for geographical data is based on geographical distance. The location of two entities is considered to be similar if they are less than $100 \mathrm{~km}$ apart. The match function is presented in Algorithm 4 . 


\begin{tabular}{|c|c|}
\hline Parameter & Value \\
\hline ID & 985 \\
\hline Name & Rok Vrabič \\
\hline URLs & (redacted) \\
\hline Keywords & $\begin{array}{l}\text { mathematica; robotics; manufacturing systems; complex- } \\
\text { ity theory; network analysis; mechatronics; production en- } \\
\text { gineering; network theory; control theory; mobile robotics; } \\
\text { programming; computer science; economics; engineering; } \\
\text { data mining; computing in mathematics, natural science, } \\
\text { engineering and medicine; artificial intelligence; experi- } \\
\text { mental economics; mechanical engineering; engineering ed- } \\
\text { ucation; manufacturing engineering }\end{array}$ \\
\hline Latitude & 46.046 \\
\hline Longitude & 14.499 \\
\hline Country & Slovenia \\
\hline
\end{tabular}

Table 1: The profile of one of the authors of the paper.

If the initial node information and the profile are sufficiently similar, the profile is determined to belong to the node. The node information is in turn updated and stored as shown in Algorithm 5.

\subsection{Step 5: Identification of connections}

The last step is the analysis of the content through which the node's connections are identified. The content analysis is based on parsing of the website's structure for the structured sources and on identification of URL links to external websites for the unstructured ones. The connections are saved along with their type. If the connections link to nodes that are not yet in the database, the new nodes are created and are considered for the next cycle of the network-identification procedure as nodes with incomplete information. The algorithm for checking whether the node is already in the 


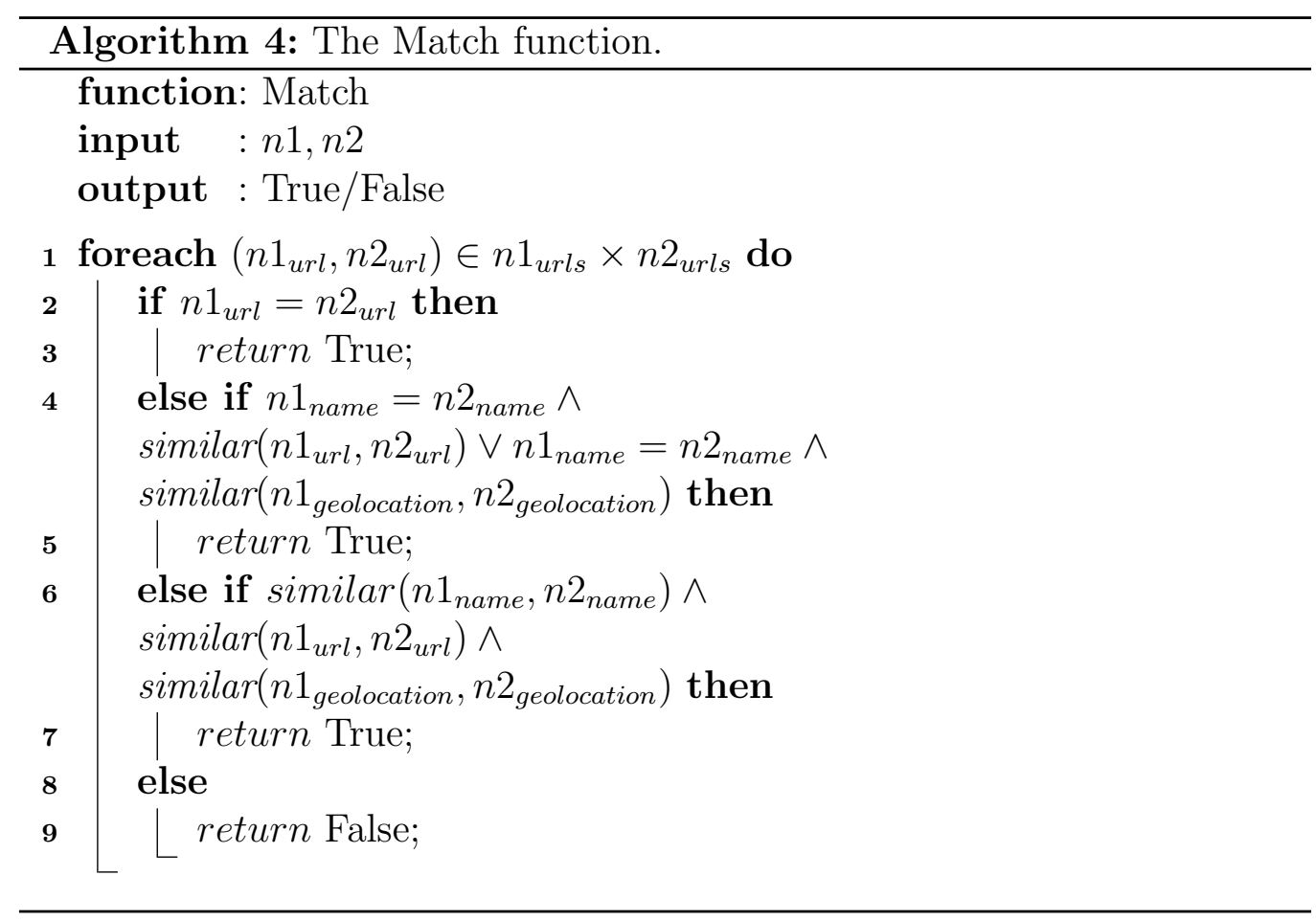

database uses the match function presented in Algorithm 4 and is presented in Algorithm 6.

The data is represented as a network in which the nodes are tagged by type, domain, extracted keywords, and geographic location. The connections are tagged with the source, the target, and the type of connection, e.g., 'website link' or 'follower' on a social network. The procedure for adding the nodes and connections to the network is presented in Algorithm 7 .

Example networks, obtained in a breadth-first manner during the subsequent cycles of the network-identification process, are shown in Fig. 3 . Starting from two seed nodes representing companies, several connections between them are identified in two cycles. The network undergoes an explosive growth in size with each cycle. Starting with two nodes, if each node 

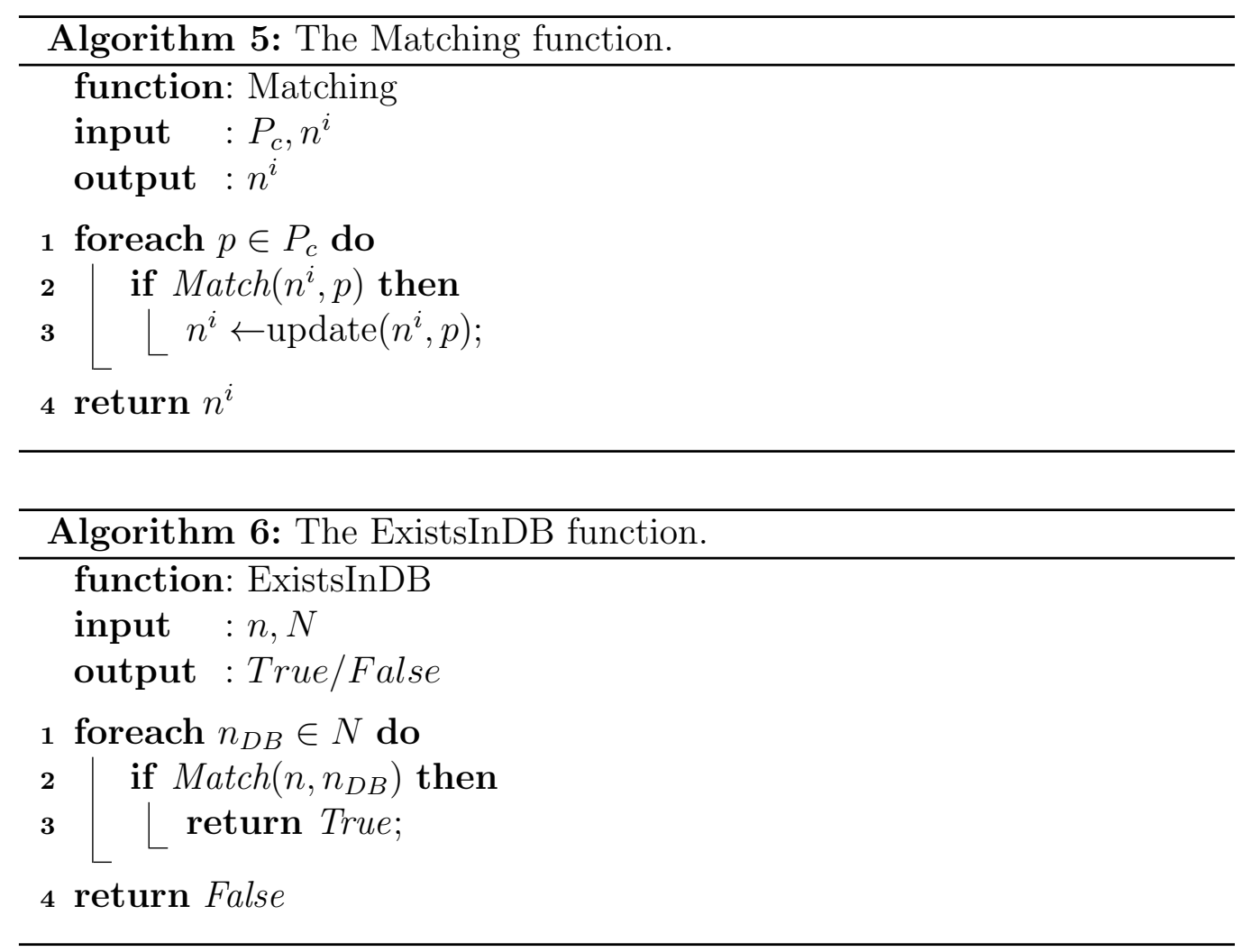

has approximately 10 connections, on average, several hundreds of nodes can be identified after only two cycles. However, this growth of the search space can be mitigated by using appropriate heuristics. In the presented approach, new connections are sought only for the nodes from the production domain. This makes possible a significant saving in the search time with a minimal loss in data value.

In principle, the described network identification procedure could be done manually, by analysing the websites, following the links, and writing down the data. In order to speed up the process by automating the procedure, the algorithms are implemented in the Python programming language. 


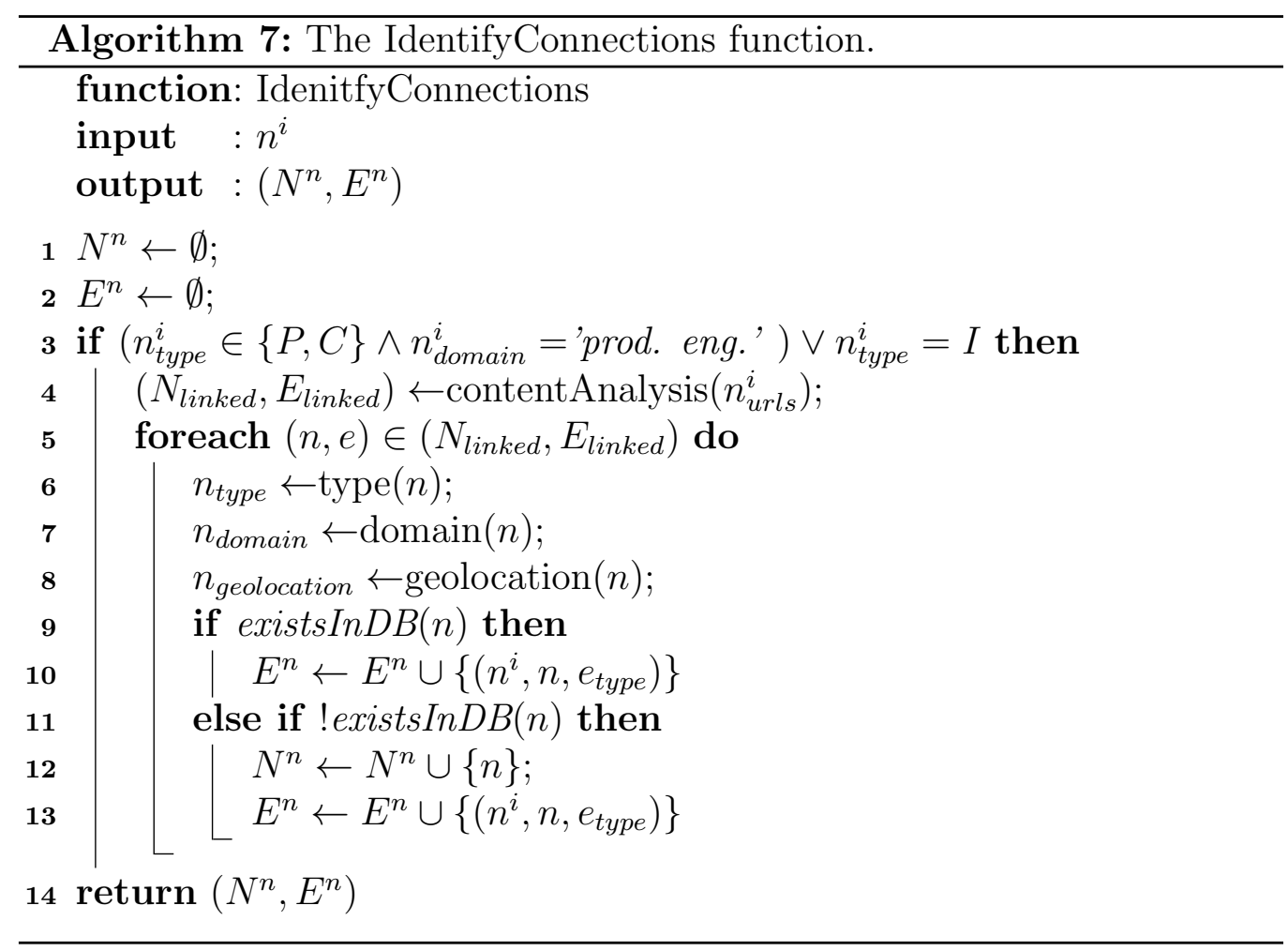

\section{Evaluation of the approach}

The approach is evaluated for the case of the automotive sector. The main reasons for this are that: (1) the relationships between companies in the automotive sector are well documented, which should allow for an interpretation of the results; (2) the automotive domain is large in the sense that the described approach should demonstrate its value; and (3) the companies in the automotive sector are well connected, forming networks with many-to-many relationships between OEMs and suppliers. Even though a quantitative validation of the approach is challenging because of the lack of reference data about the interactions of mixed communities of people, companies, and institutions, it is expected that the results will qualitatively 


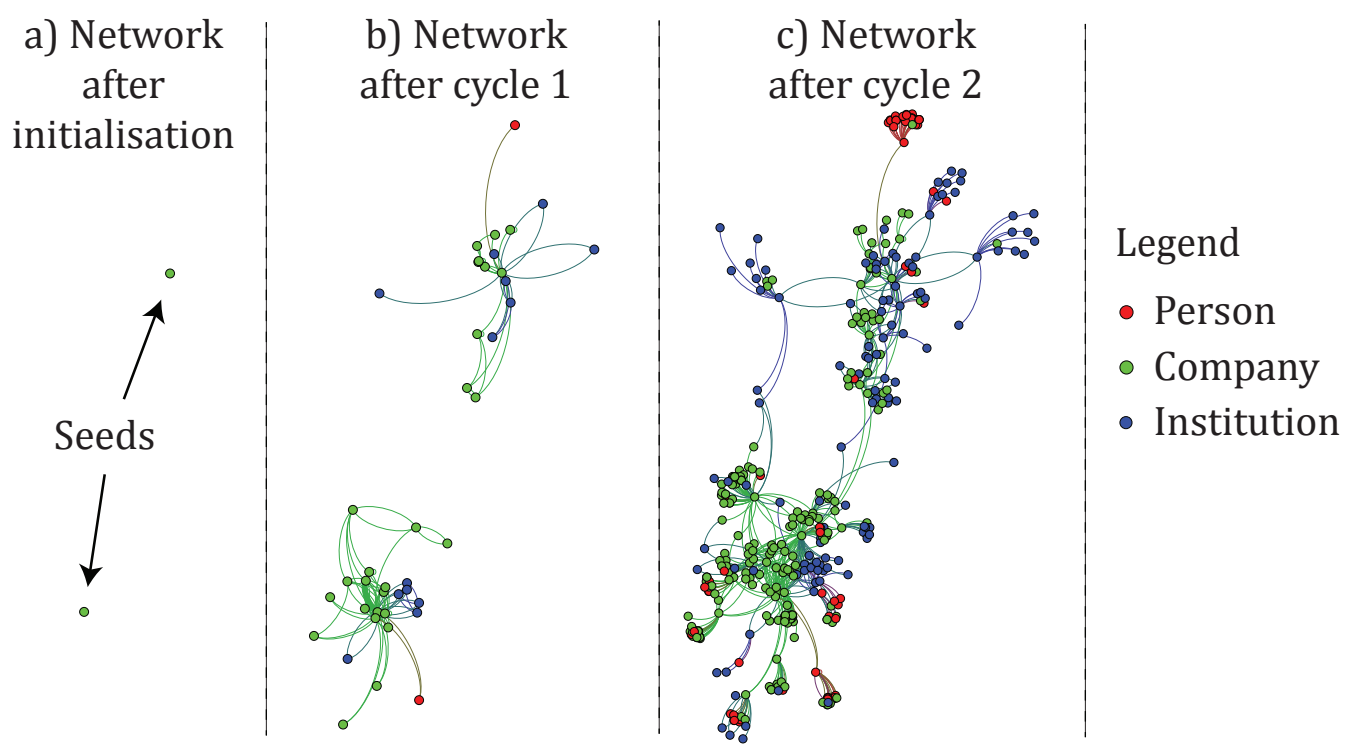

Figure 3: Example of the result of the network-identification process after a) initialisation, b) 1 , and c) 2 cycles.

describe the important features of the real-world network. The case study includes 7 OEMs and 7 suppliers as the initial seeds. The depth of the search, i.e., the number of cycles, is set to two, meaning that connections of the initial seeds as well as their connections are identified. All the data sources, the web crawl, the professional social network, and the scientific social network are used. The overview of the resulting network is presented in Fig. 4.

The results show that the method adequately captures the large-scale network structure of the automotive domain as exhibited by the groupings of the manufacturers, such as large consortia, which are easily identifiable within the network structure. For example, the Italian OEMs highlighted in Fig. 4 consist of three sports-car brands and an OEM, which is a part of a larger consortium with US manufacturers. A closer look at the sportscar manufacturers shown in Fig. 5 shows that they are closely linked with 


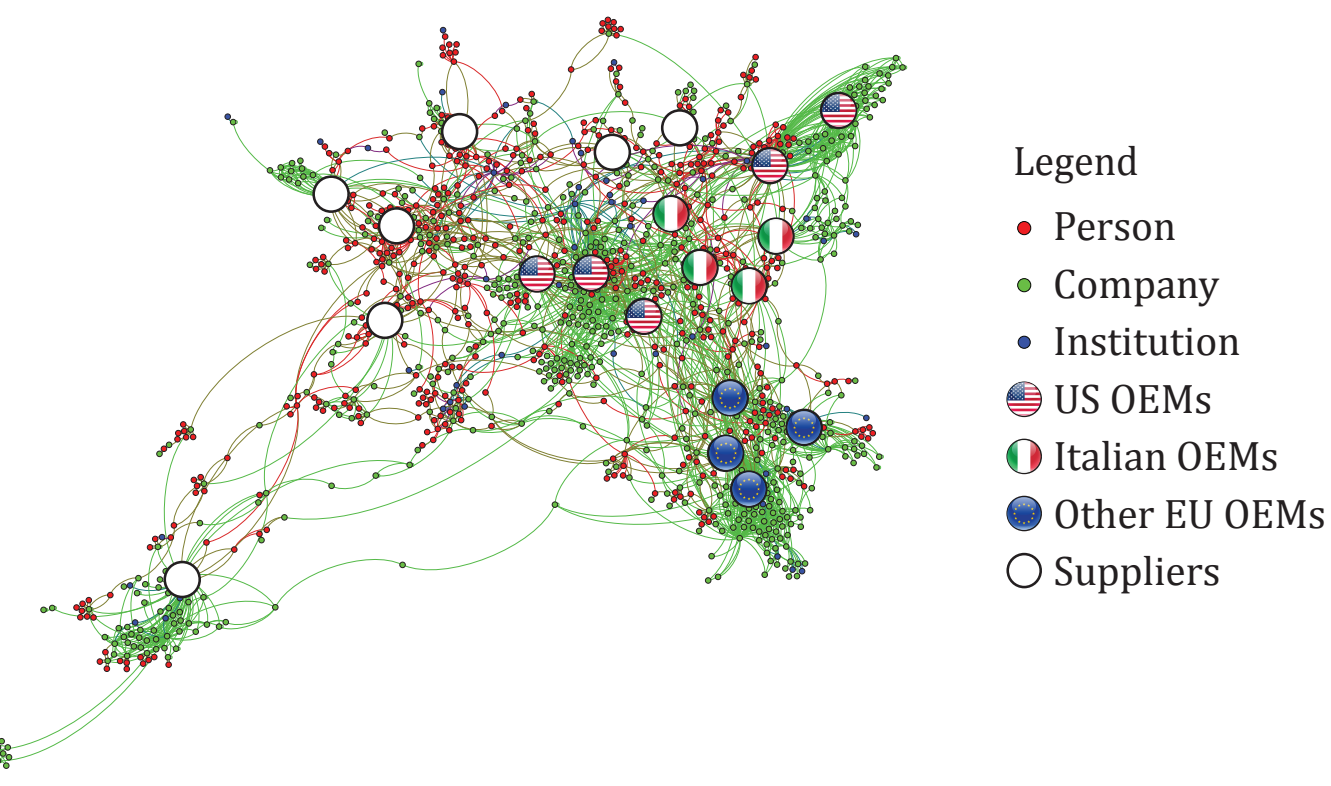

Figure 4: Network of the automotive domain; selected interesting nodes highlighted.

the same brakes and lights suppliers which is well aligned with real-world observations.

The automotive sector also exhibits strong regional patterns (Sturgeon et al., 2008; Mariel and Minner, 2015: Häntsch and Huchzermeier, 2016). The US manufacturers are grouped near the centre of the network. Similarly, the EU manufacturers are grouped in the bottom right of Fig. 4. The suppliers are typically smaller and more peripheral to the network. The supplier in the bottom left is the most remote because it is part of a large, but geographically distant, Japanese conglomerate.

The results, overlayed on the world map, are shown in Fig. 6. The network is well connected. It can be observed that out of the manufacturers under considerations, the European ones exhibit more connections with South America than the US ones, which is in line with expectations. 


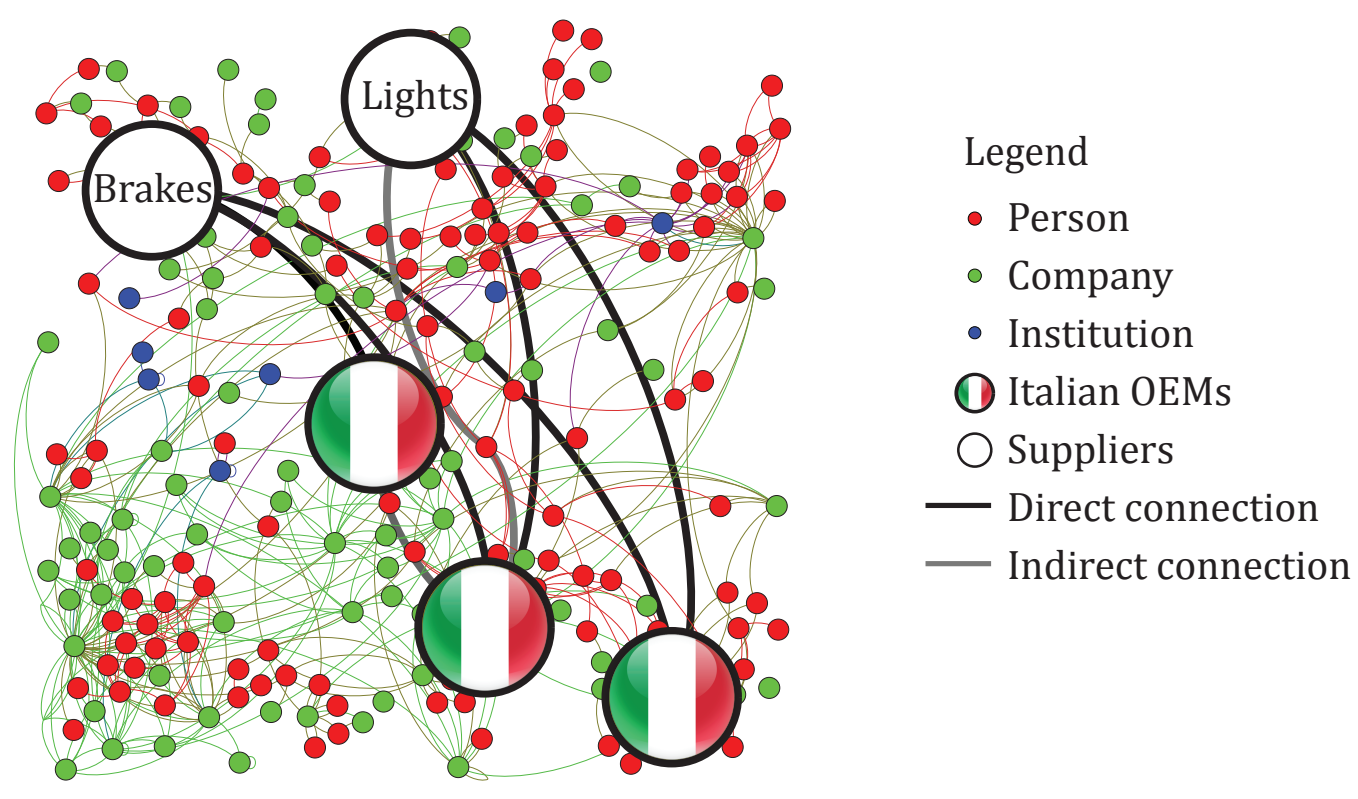

Figure 5: The subnetwork of the Italian sports-car manufacturers.

Because the initial seeds are all companies, they also represent the largest part of the resulting network. The overall network structure consists of $30.8 \%$ of companies, $6.3 \%$ of institutions, and $5.0 \%$ of people. The rest is structured as follows: $13.0 \%$ of companies from other domains, $6.1 \%$ of people from other domains, and $38.8 \%$ of other nodes. The connections are dominated by two sources: $56 \%$ come from the web crawl and $44 \%$ come from the analysis of the professional social network. The scientific social network analysis accounts for less than $1 \%$ of the connections. This implies that the automotive manufacturers do not publicly advertise their connections with the institutions. The OEMs have, on average, more connections than the suppliers and are significantly more central to the network, which confirms the real-world observations (Kito and Ueda, 2014). The network statistics are presented in Table 2 , which shows the degree (average number of connections), the close- 


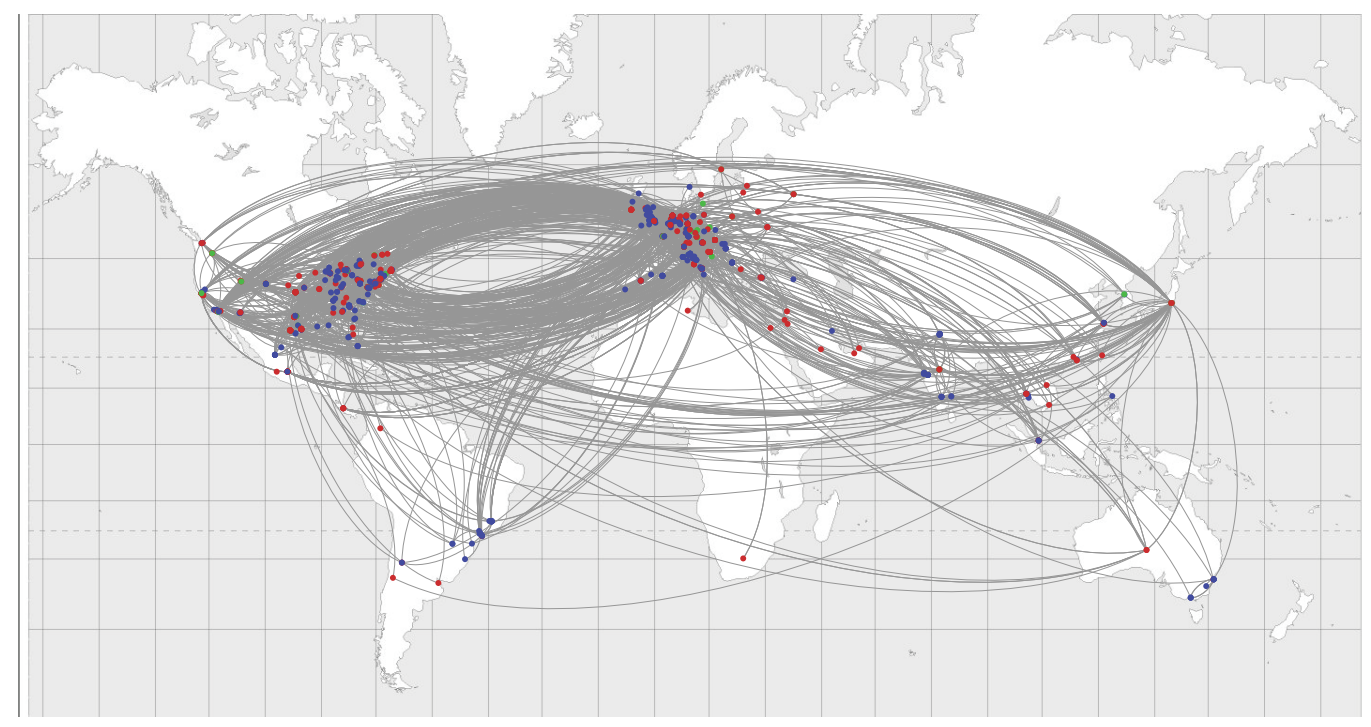

Figure 6: The results overlayed on the world map.

ness (average normalized distance between the nodes), and the betweenness (the normalized number of times a node acts as a bridge along the shortest path between two other nodes) centralities.

\begin{tabular}{|l|r|r|r|}
\hline Node type & Degree & Closeness & Betweenness \\
\hline OEMs & $87.7 \pm 27.3$ & $.264 \pm .027$ & $.0465 \pm .0361$ \\
Suppliers & $34.7 \pm 15.3$ & $.200 \pm .016$ & $.0229 \pm .0176$ \\
\hline
\end{tabular}

Table 2: The degree, the normalised closeness, and the normalised betweenness centralities of the nodes with respect to their type; mean \pm standard deviation.

The method is shown to accurately and qualitatively describe the domain in question, as it confirms all of the following intuitive assumptions: (1) the company groupings of the identified network correspond to the ones expected based on real-world observations (Sturgeon et al., 2008), (2) the suppliers are less connected and more peripheral to the network (Kito and Ueda, 2014), 
and (3) the network is well connected with a large main component.

\section{Demonstration of use: Seach for skills and expertise within the network}

The resulting networks describe people, companies, and institutions in the domain of production along with their interconnections and additional data, such as geolocation and keywords. The keywords describe the data available through public profiles on social networks, e.g. data about a person's field of interest, skills, and expertise (Sun et al., 2015). This section demonstrates how the resulting network data can be used for skill profiling and search for expertise and showcases the potential of the approach.

The presented network identification procedure is applied to the data of one of the leading research communities from the domain of production engineering, CIRP (Santochi, 2013). The community consist of more than 600 members and is particularly interesting because even though it is primarily academic it includes more than 100 members from industry.

The goal of the case study is to demonstrate how the presented approach can be used to look for particular skills and expertise within a network, based on the related keywords acquired from the social networks. The approach can be used to not only identify relevant nodes, but, in addition, to identify the connections between the nodes that can be used to facilitate the networking process. This procedure highlights the unique aspects of the approach and the particular nature of the information obtainable from the social networks.

The workflow of the study is shown in Fig. 7 and consists of the following:

a) The definition of the initial seeds, the domain of interest, and the clas- 

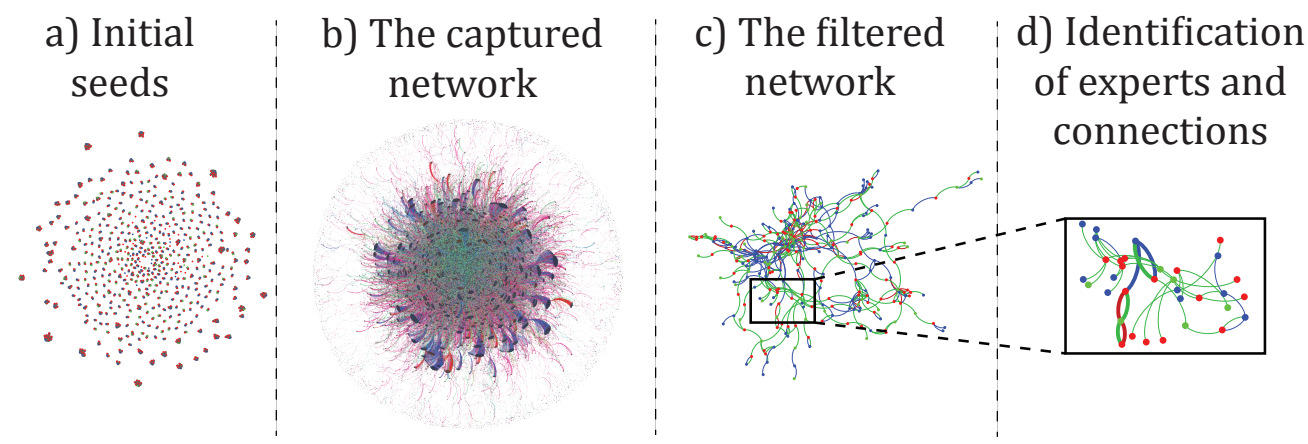

Figure 7: The workflow of the case study.

sification of the corresponding expertises into fields. The classification is neccessary because the expertises listed on the social networks can be very narrow - sometimes even unique to a person. The classification into broader fields facilitates the filtering of the results and improves the robustness of the search because an expertise can be matched through several, similar keywords.

b) The acquisition of the data using the presented method to capture the structure of the network and, for each individual node, keywords related to their skills and expertise.

c) Filtering of the acquired data to identify only relevant connections that can in turn be used to facilitate the networking process. This step needs to be performed to reduce the amount of data.

d) Applying the classification of the expertises into fields to the filtered network, which results in the identification of the nodes posessing the required expertise and the relevant connections within the network. 


\subsection{Classification of expertise}

The chosen community is well suited for the described procedure. Most of the community's members publish scientific papers on various topics of production engineering. The community is structured into 10 fields within the domain of production engineering, as shown in Table 3 .

\begin{tabular}{|l|l|}
\hline Acronym & Field \\
\hline A & Life Cycle Engineering and Assembly \\
C & Cutting Processes \\
Dn & Design \\
E & Electro-Physical and Chemical Processes \\
F & Forming \\
G & Abrasive Processes \\
M & Machines \\
O & Production Systems and Organisations \\
P & Precision Engineering and Metrology \\
S & Surfaces \\
\hline
\end{tabular}

Table 3: Fields in the domain of production engineering according to CIRP.

The information for each member includes the primary field of interest. Together with the data from their scientific papers, this can be used to create a classifier associating the keywords with the fields. Multinomial naïve Bayes classifier, which is well suited for text classification, is used.

The classifier is trained on 2050 scientific papers, 205 from each field. Titles, abstracts, and keywords are used as the input, while the primary field of the paper is considered to be the output category. Stop words are removed and stemming is used to improve the accuracy. The classifier is validated using stratified 10-fold cross-validation. $81.78 \%$ accuracy is achieved.

Table 4 shows five most significant keywords for each of the fields. 


\begin{tabular}{|l|r|r|r|r|r|}
\hline Field & Keyword1 & Keyword2 & Keyword3 & Keyword4 & Keyword5 \\
\hline $\mathrm{A}$ & assembly & product & process & design & manufacturing \\
$\mathrm{C}$ & cutting & tool & machining & surface & milling \\
$\mathrm{Dn}$ & design & product & process & method & model \\
$\mathrm{E}$ & laser & machining & edm & process & micro \\
$\mathrm{F}$ & forming & process & sheet & metal & material \\
$\mathrm{G}$ & grinding & process & surface & wheel & abrasive \\
$\mathrm{M}$ & machine & tool & machining & control & cutting \\
$\mathrm{O}$ & production & manufacturing & system & planning & process \\
$\mathrm{P}$ & measurement & measuring & machine & metrology & optical \\
$\mathrm{S}$ & surface & process & micro & polishing & roughness \\
\hline
\end{tabular}

Table 4: The five most significant keywords for each field.

The result in Table 4 is in line with the expectations. Even though some keywords such as process appear in different fields, the fields are logically separated with the most significant keyword meaningfully describing each field.

Table 5 illustrates example results of the classification. The results show that the classifier which is trained on the data obtained from the scientific papers is useful for the classification of the keywords obtained from the social networks.

\subsection{Data acquisition}

The public information on community's website includes information about 658 members from 440 different companies and institutions at the time of the study. For each member the full name, the organisation with which they are associated, the country, and optionally the company website URL address are provided. This incomplete information is used for the generation of the seed nodes and initial connections. The initial network is shown in Fig. 7 . 


\begin{tabular}{|l|l|}
\hline Keywords & $\begin{array}{l}\text { Top prediction(s) } \\
\text { (Confidence) }\end{array}$ \\
\hline $\begin{array}{l}\text { knowledge-based systems; process development; } \\
\text { product development; mechanical design; supply } \\
\text { chain; engineering design; process design; produc- } \\
\text { tion planning; mechanical engineering design; cad }\end{array}$ & Dn (99.98\%) \\
\hline cnc; cad; cam & $\mathrm{M}(76.94 \%)$ \\
\hline $\begin{array}{l}\text { fuzzy control; robotics; fuzzy logic; manufacturing } \\
\text { engineering; neuro-fuzzy }\end{array}$ & $\mathrm{A}(79.44 \%)$ \\
\hline $\begin{array}{l}\text { machinery; rotor dynamics; optimization; machine } \\
\text { condition monitoring; optical measurement; on } \\
\text { machine measurement; solid mechanics; manufac- } \\
\text { turing process; digital manufacturing }\end{array}$ & $\mathrm{P}(42.06 \%) ;$ \\
\hline $\begin{array}{l}\text { complex systems; manufacturing systems; machin- } \\
\text { ing; cnc machining; manufacturing process me- } \\
\text { chanics; cad; mechanical processes; production en- } \\
\text { gineering; manufacturing; operations; production; }\end{array}$ & $\mathrm{O} \mathrm{(99.81 \% )}$ \\
$\begin{array}{l}\text { industry; production systems; simulation exper- } \\
\text { iments; engineering design; engineering; control } \\
\text { systems engineering; computer engineering; man- }\end{array}$ & \\
ufacturing engineering & \\
\hline
\end{tabular}

Table 5: Illustration of top classifier predictions.

The number of cycles of the network identification procedure is set to one, meaning that only the initial seeds' connections are identified. All three data sources are used. The data aquisition for this case took approximately 300 hours on a single PC running the described algorithms. The resulting network, shown in Fig. 7b, consists of approx. 50,000 nodes and 65,000 edges of all types and domains. 


\subsection{Reducing the amount of information}

The information about the entire network, shown in Fig. 7b, is difficult to use due to its size. The number of connections grows with the number of nodes as each new node introduces several new connections. The number of introduced connections depends on the data source and on the connectedness of the node, but, since for example each person on a social network is connected to a finite number of other people, the growth of connections is approximately linear with the network size. However, analysis of connections has to take into account several connections at the same time which introduces combinatorial and, in turn, computational complexity.

A reasonable approach is to define what is relevant information and then filter the network accordingly. In this case the network is filtered to include only the seed nodes and relevant connections between them. These connections can be direct or indirect - through other nodes. For this example, the

relevant connections, in form (node type)-(node type)-etc., are considered to be the following:

1. (company/institution)-(company/institution)

2. (company/institution)-(person)

3. (person)-(person)

4. (company/institution)-(person)-(company/institution)

5. (company/institution)-(person)-(person)-(company/institution)

The filtered network consists of 102 people, 218 companies and institutions, and 475 connections. Its main component is shown in Fig. 8. Not all nodes are a part of the main component, meaning that the network is 
not fully connected. The figure illustrates the size and the structure of the network. The density of the network varies, which reflects the characteristics of the community, mainly, that certain members and institutions are more closely involved with the community (attending conferences, publishing papers, participating in projects, etc.) than others.

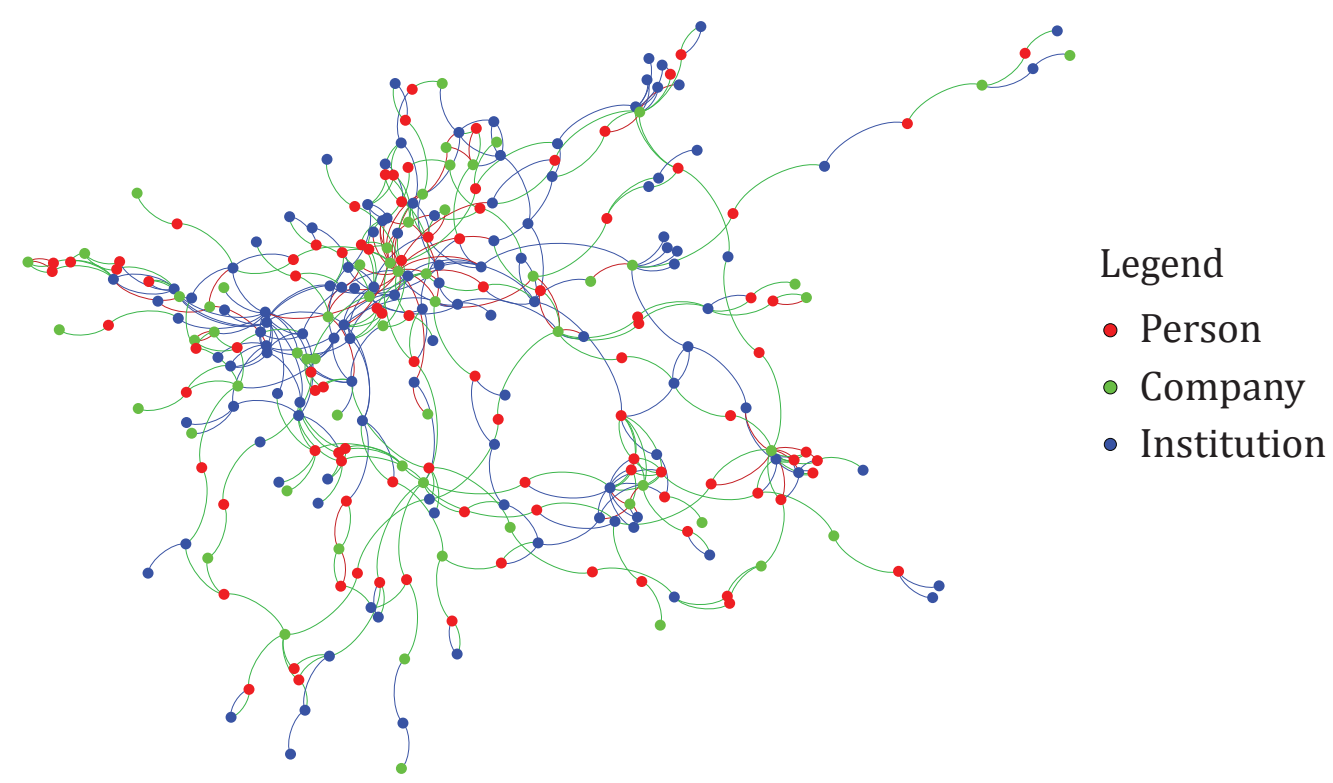

Figure 8: The filtered network in which only the relevant connections are kept.

\subsection{Identification of expertise}

The identification of expertise is illustrated in a case of an aerospace company covering commercial aeronautics, defence, and space markets. The company is looking for expertise in the field of material science. Applying the presented approach, the company is able to retrieve the filtered network. Using any network exploration tool, connections depicted in Fig. 9 can be quickly extracted. 


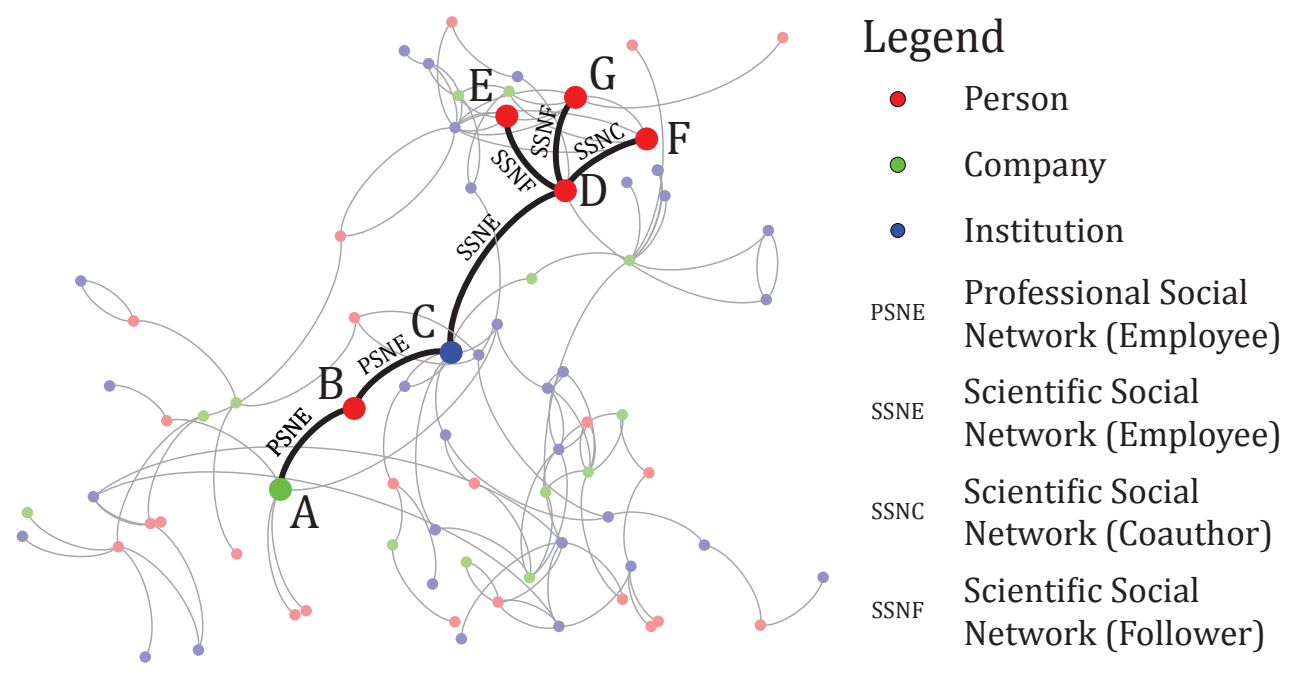

Figure 9: Identification of expertise within the filtered network. Node markings are explained in text.

An interesting link is discovered. An employee (node B) of the company (node A) also works for a technical university (node C). The source of both links is the professional social network. The employee is a member of the scientific community and is classified into field O with 99,9\% confidence. His keywords include "leadership", "project management", "organisational development", and "game theory", among others. Through the scientific social network, another person (node D) is also identified to work for the same technical university and is also a member of the same scientific community. This implies that the two know each other or are at least able to contact each other easily. Person D is classified into fields E (65.3\% confidence) and S (15.7\% confidence). His keywords include "surface properties", "materials", and "powder metallurgy", among others.

Person D is, in turn, connected to people E, F, and G through coauthorship of papers and the "follower" relation. This information is ex- 
tracted from the scientific social network. Their keywords include "mathematical physics" and "materials physics" (person E), "metal nanoparticles" and "materials engineering" (person F), and "functionally graded material", "grinding", and "abrasive machining" (person G). Clearly, the expertise of people $\mathrm{E}$ and $\mathrm{F}$ is interesting for the company.

This illustrative case, based on real data, demonstrates that new insights are obtained by combining the information from the different sources and that the classification of the expertise can be used not only as a filtering tool but also a search heuristic. It also shows how the presented approach goes beyond large-scale extraction of the publicly available data by demonstrating that new information can be gained by combining the data from different sources.

\subsection{Discussion}

The case study demonstrates a potential use of the approach, i.e., how the information collected from the unstructured and structured publicly available data can be used to give an insight into the structure of a community.

The extraction of relevant structures addresses the problem of the large size of the extracted data. The approach shown in the example filters the network to only retain nodes connected by specific types of connections. Since the extracted information includes detailed node information in addition to the connection types, extensions of the presented example could take that into account to provide precise filtering of the extracted data.

An important aspect of the approach is that it enables an analysis of the skills and the expertise associated with the nodes. As illustrated by the case, it is useful to develop advanced methods of analysis in order to tackle the 
size and the complexity of the data. The classification of keywords denoting the skills and the expertise of individuals into fields proves useful for filtering of the large amounts of data as well as for providing a heuristic during the combinatorially complex search for paths between the nodes.

\section{Conclusions}

The paper demonstrates the use of the advanced computational methods and data analytics for identifying networks of individuals, companies, and institutions from publicly available, structured and unstructured, heterogeneous data. An approach is developed for identification and evaluation of networks from online publicly available data. The feasibility of the approach is studied for the case of the automotive sector, where it is shown that the results are in line with the expectations and with other investigations relating to the structure of real-world networks. The case study of the production engineering community shows how the approach can be used to analyse the community's structure and search for expertise.

Even though the presented network-identification procedure is proven to be feasible, it is limited in depth, because of the rapidly increasing search space and the limitation of the application programming interfaces (API) of the services such as geolocation, translation, and search. For example, the data acquisition for the presented studies took several hundreds of hours. This limits the real-time applicability of the method at this stage, but could be improved in the future.

The approach has the potential to be used for the modelling and analysis of various forms of collaboration between and within businesses and individ- 
uals. As a tool, it could be used for the purposes of strategic networking, to facilitate the creation of project consortia, to identify competitors and other stakeholders in a certain domain, to pinpoint communication channels, to search for expertise, or to identify organisational and social structures within organisations. Future extensions could include more public information sources, such as news stories, as well as private information sources such as company communication and email networks. The Big Data approach taken in this study could be improved with the use of stronger machine learning approaches and massively-parallel computing methods.

Lastly, the issue of privacy must be thoroughly addressed in these types of studies. Even though the information presented here is gathered from public sources and could be, in principle, acquired manually, any future studies should be careful to handle and present any individual's information in an ethically appropriate way.

\section{Acknowledgements}

This work was partially supported by the Ministry of Higher Education, Science and Technology of the Republic of Slovenia, Grant number 1000-150510, and by the Slovenian Research Agency, Grant number P2-0270.

\section{References}

Acar, Y., Atadeniz, S.N., 2015. Comparison of integrated and local planning approaches for the supply network of a globallydispersed enterprise. International Journal of Production Economics 167, 204 -19. URL: http://www.sciencedirect.com/science/ 
article/pii/S0925527315001802, doi/http://dx.doi.org/10.1016/j.

ijpe.2015.05.028.

Alexa, 2017. Actionable analytics for the web. URL: http://www.alexa. com. accessed May 2017.

Becker, T., Meyer, M., Windt, K., 2014. A manufacturing systems network model for the evaluation of complex manufacturing systems. International Journal of Productivity and Performance Management 63, $324-40$. URL: http://dx.doi.org/ 10.1108/IJPPM-03-2013-0047, doi:10.1108/IJPPM-03-2013-0047, arXiv:http://dx.doi.org/10.1108/IJPPM-03-2013-0047.

Chae, B.K., 2015. Insights from hashtag supplychain and Twitter analytics: Considering Twitter and Twitter data for supply chain practice and research. International Journal of Production Economics 165, 247 -59. URL: http://www.sciencedirect.com/science/ article/pii/S0925527314004319, doi:http://dx.doi.org/10.1016/j. ijpe.2014.12.037.

Chen, M., Mao, S., Liu, Y., 2014. Big data: A survey. Mobile Networks and Applications 19, 171-209.

Häntsch, M., Huchzermeier, A., 2016. Transparency of risk for global and complex network decisions in the automotive industry. International Journal of Production Economics 175, 81 - 95.

Hitzler, P., Janowicz, K., 2013. Linked data, big data, and the 4th paradigm. Semantic Web 4, $233-5$. 
Hu, H., Wen, Y., Chua, T.S., Li, X., 2014. Toward scalable systems for big data analytics: a technology tutorial. Access, IEEE 2, 652-87.

IQS, 2016. Manufacturer directory. URL: http://www.iqsdirectory.com. accessed May 2017.

Kito, T., New, S., Ueda, K., 2015. How automobile parts supply network structures may reflect the diversity of product characteristics and suppliers' production strategies. CIRP Annals - Manufacturing Technology 64, 423 -6. URL: http://www.sciencedirect.com/science/ article/pii/S0007850615001250, doi:http://dx.doi.org/10.1016/j. cirp.2015.04.117.

Kito, T., Ueda, K., 2014. The implications of automobile parts supply network structures: A complex network approach. CIRP AnnalsManufacturing Technology 63, $393-6$.

Laney, D., 2001. 3-D data management: Controlling data volume, variety and velocity. META Group File 949.

Lanza, G., Moser, R., 2014. Multi-objective optimization of global manufacturing networks taking into account multi-dimensional uncertainty. CIRP Annals - Manufacturing Technology 63, 397 - 400. URL: http:// Www.sciencedirect.com/science/article/pii/S000785061400119X, doi:http://dx.doi.org/10.1016/j.cirp.2014.03.116.

Mariel, K., Minner, S., 2015. Strategic capacity planning in automotive production networks under duties and duty drawbacks. International Journal of Production Economics 170, 687 - 700. 
Mourtzis, D., Doukas, M., Milas, N., 2016a. A knowledge-based social networking app for collaborative problem-solving in manufacturing. Manufacturing Letters $10,1-5$.

Mourtzis, D., Doukas, M., Psarommatis, F., 2013. Design and operation of manufacturing networks for mass customisation. CIRP Annals - Manufacturing Technology 62, 467 -70. URL: http:// www.sciencedirect.com/science/article/pii/S0007850613001273, doi:http://dx.doi.org/10.1016/j.cirp.2013.03.126.

Mourtzis, D., Vlachou, E., Milas, N., 2016b. Industrial big data as a result of IoT adoption in manufacturing. Procedia CIRP 55, 290-5.

Neto, J.Q.F., Bloemhof, J., Corbett, C., 2016. Market prices of remanufactured, used and new items: Evidence from eBay. International Journal of Production Economics 171, Part 3, 371 -80. URL: http:// www.sciencedirect.com/science/article/pii/S0925527315000407, doi:http://dx.doi.org/10.1016/j.ijpe.2015.02.006. operations Management and Production Research Using Secondary Data.

Papakostas, N., Georgoulias, K., Koukas, S., 2013. A novel platform for designing and evaluating dynamic manufacturing networks. CIRP Annals - Manufacturing Technology 62, 495 -8. URL: http:// www.sciencedirect.com/science/article/pii/S0007850613001121, doi:http://dx.doi.org/10.1016/j.cirp.2013.03.111.

Putnik, G.D., Škulj, G., Vrabič, R., Varela, L., Butala, P., 2015. Simulation study of large production network robustness 
in uncertain environment. CIRP Annals - Manufacturing Technology 64, 439 -42. URL: http://www.sciencedirect.com/science/ article/pii/S0007850615001262, doi:http://dx.doi.org/10.1016/j. cirp.2015.04.118.

Qi, X., Davison, B.D., 2009. Web page classification: Features and algorithms. ACM computing surveys (CSUR) 41, 12.

Rajput, A., Aharwal, R.P., Dubey, M., Saxena, S., Raghuvanshi, M., 2011. J48 and JRIP rules for e-governance data. International Journal of Computer Science and Security (IJCSS) 5, 201.

Santochi, M., 2013. CIRP: A unique academy in production engineering. Procedia CIRP 12, 3-6.

Škulj, G., Vrabič, R., Butala, P., 2014. Experimental study of work system networking in production environment. CIRP Annals - Manufacturing Technology 63, 401 -4. URL: http://www.sciencedirect.com/science/ article/pii/S0007850614001188, doi:http://dx.doi.org/10.1016/j. cirp.2014.03.115.

Sturgeon, T., Van Biesebroeck, J., Gereffi, G., 2008. Value chains, networks and clusters: reframing the global automotive industry. Journal of economic geography 8, 297-321.

Sun, J., Xu, W., Ma, J., Sun, J., 2015. Leverage RAF to find domain experts on research social network services: A big data analytics methodology with MapReduce framework. International Journal of Production Economics 165, 185-93. 
Tan, K.H., Zhan, Y., Ji, G., Ye, F., Chang, C., 2015. Harvesting big data to enhance supply chain innovation capabilities: An analytic infrastructure based on deduction graph. International Journal of Production Economics $165,223-33$.

Wamba, S.F., Akter, S., Edwards, A., Chopin, G., Gnanzou, D., 2015. How 'big data' can make big impact: Findings from a systematic review and a longitudinal case study. International Journal of Production Economics 165, 234 -46. URL: http://wWw.sciencedirect.com/science/ article/pii/S0925527314004253, doi:http://dx.doi.org/10.1016/j. ijpe.2014.12.031.

Wiendahl, H.P., Lutz, S., 2002. Production in networks. CIRP Annals - Manufacturing Technology 51, 573 -86. URL: http://www. sciencedirect.com/science/article/pii/S0007850607617016, doi:http://dx.doi.org/10.1016/S0007-8506(07)61701-6.

Witten, I.H., Frank, E., Hall, M.A., Pal, C.J., 2016. Data Mining: Practical machine learning tools and techniques. Morgan Kaufmann.

Zhong, R.Y., Huang, G.Q., Lan, S., Dai, Q., Chen, X., Zhang, T., 2015. A big data approach for logistics trajectory discovery from RFIDenabled production data. International Journal of Production Economics 165, 260 -72. URL: http://www.sciencedirect.com/science/ article/pii/S0925527315000481, doi:http://dx.doi.org/10.1016/j. ijpe.2015.02.014. 